\title{
Real-time Collaborative Tracking for Underwater Networked Systems
}

\author{
D. Mirza ${ }^{a}$, P. Naughton ${ }^{b}$, C. Schurgers ${ }^{\mathrm{a}}$, R. Kastner ${ }^{\mathrm{a}}$ \\ ${ }^{a}$ Department of Computer Science and Engineering, University of California, San Diego, \\ 9450 Gilman Drive, La Jolla, California 92092 \\ ${ }^{b}$ Center of Interdisciplinary Science for Art, Architecture and Archaeology (CISA3), \\ Qualcomm Institute, Calit2, University of California, San Diego
}

\begin{abstract}
Localization is a crucial requirement for mobile underwater systems. Real-time position information is needed for control and navigation of underwater vehicles, in early warning systems and for certain routing protocols. Past research has shown that the localization accuracy of networked underwater systems can be significantly improved using inter-vehicle collaboration. More specifically the Maximum Likelihood (ML) position estimates of a mobile collective can be computed from measurements of relative positions and motion, albeit in a non-real-time fashion. In this work we extend this solution to compute the position estimates of a network in real-time and in a distributed fashion. We first describe a centralized approach to identify key factors that fundamentally limit the performance of a real-time solution. Using the centralized approach as a benchmark, we arrive at a real-time distributed solution that additionally computes the location of vehicles using information obtained locally by them. We address practical considerations in the implementation of our algorithm and propose solutions to mitigate computational errors. With this proposed implementation, we provide insight on how to appropriately plan a deployment of nodes when collaborative tracking is to be utilized. Lastly, we shed light on situations where implementing collaborative tracking can hinder the localization
\end{abstract}

Email addresses: dimirza@eng.ucsd.edu (D. Mirza), pnaughto@eng.ucsd.edu (P. Naughton), cschurgers@ucsd.edu (C. Schurgers), kastner@cs.ucsd.edu (R. Kastner)

Preprint submitted to Journal of Ad Hoc Networks

October 12, 2014 
performance of the network so that these can be avoided.

Keywords: Underwater Networks, Real-time Tracking, Acoustic Networks

\section{Introduction}

There has been a growing interest in operating groups of autonomous underwater vehicles in a networked fashion, using short to medium range acoustic modems [1. Location information is critical to such networked mobile underwa-

5 ter systems for correctly annotating data samples, control and navigation and in certain data routing protocols. However, since GPS is not available underwater, the position of vehicles has to be estimated over time.

Most existing solutions based on Kalman or particle filters are designed for tracking vehicles individually [2] 3]. This is achieved by combining measurements of relative position with respect to known landmarks or beacons together with estimates of the vehicle's motion obtained from on board sensors. These non-collaborative tracking methods perform poorly in underwater networks where vehicles have short to medium acoustic communication range. This is due to the fact that a vehicle may not be within the communication range of a sufficient number of beacons or it may move out of range of beacons from time to time. A solution to this problem involves using additional measurements for localization, which can be obtained from communication between vehicles. Such a collaborative approach has been employed by a number of localization techniques such as multi-dimensional scaling and iterative multi-lateration. However, these techniques have been proposed for stationary networks. They typically use relative position information (distance, proximity or direction) as geometric constraints on the unknown location of devices. Since devices are stationary, the constraints can be considered concurrently to solve for the unknown locations. In principle these algorithms can be extended to mobile underwater networks by localizing time snapshots of the network as suggested in [4] 5] 6]. However this requires measurements of relative position to be obtained concurrently. Further, position estimates for each snapshot must 
be computed before there is significant displacement in the vehicle positions. In reality, due to practical communication constraints, the use of such techniques for underwater networks would be severely limited when deployments are sparse and vehicles are moving.

Our prior work has shown that in sparse mobile underwater networks, localization accuracy can be significantly improved when vehicles collaborate [7, compared to both individual tracking and collaborative static localization. We proposed a collaborative solution that tracks a group of vehicles as a collective by jointly computing the trajectories of all vehicles using non-concurrent estimates of inter-node distances together with measurements of motion from on-board navigational sensors. While there exist a few collaborative tracking methods that also loosely follow the same idea, our solution is optimal as it 40 jointly computes the Maximum Likelihood (ML) positions of all vehicles over time.

The drawback of this optimal ML solution is that it is centralized and can only be applied offline, after all measurements are available at a single location. It serves applications where position information is only needed post-facto, but cannot solve the problem of real-time localization. Creating a real-time tracking solution for mobile underwater networks is a difficult task due to the power required for inter-node communication and the band limited acoustic channel of the ocean. Despite these challenges, our work in 8 modified our existing ML solution to compute positions in real-time. In this work we first propose a centralized real-time algorithm which computes all position estimates at a central location albeit in real-time. We use this solution to highlight the inherent loss in localization performance when we go from a non-real-time to a real-time solution. More practically, we propose a localized distributed solution for our real-time tracking that minimizes the communication overhead by strategically 55 sharing information between vehicles, yet achieves localization accuracies close to the best case. The essential problem that we address to this end is to determine what information to share between vehicles and when, as well as how to encode information to minimize the communication overhead. 
While the work of [8] and [7] only considered one set of trajectories in their analysis, in this work we provide additional analysis of these techniques by applying them to different motion models. We find that there are cases where the collaborative framework for localization does not perform best, but often it can alleviate many different sources of error in an underwater network and provide results that are superior to the non-collaborative case. Ultimately, we propose that careful consideration of the motion models of the vehicles as well as the positioning of the beacons must be taken into account when planning a deployment of vehicles who are collaborating to determine their position. We provide insight on how these parameters affect the location accuracy of each vehicle so that an informed decision can be made on whether or not to 70 collaboratively localize as well as how to set up a network to reduce localization error when collaborating. Before we can delve into this analysis, we will describe our prior work on non-real-time centralized tracking as well as centralized and distributed real-time tracking. This knowledge forms the basis of later analysis on deployment considerations.

\section{Related Work}

A detailed discussion of how our factor-graph framework relates to other estimation techniques that are traditionally used for underwater navigation, in robotics as well in terrestrial sensor networks can be found in our prior work [7]. A number of real-time and distributed localization techniques have been proso posed for underwater networks [4] 9 [ [5] 6]. Among these DNRL [4 and LSL [6] use multi-lateration while USP [5] applies iterative bilateration. To improve the localization accuracy, DNRL proposes the use of mobile beacons. In LSL a hierarchical (infrastructured) approach is proposed for localization [6]. These techniques essentially estimate positions for time snapshots of the network using only spatial constraints (estimates of inter-vehicle distances) and do not account for vehicle motion. The main drawback compared to our solution is that they would not work well in sparse networks and need more frequent signaling. A 
more in depth survey is available in [10]. A comparative performance evaluation of some of these schemes has been done in [11.

AUV navigation using a single mobile beacon is proposed in [2] 3. Here measurements of vehicle motion are combined with non-concurrent distance estimates to the beacon. In addition path planning is done to improve the tracking performance [3]. A collaborative solution has been proposed for underice AUV tracking where inter-vehicle estimates of distance and relative velocities (from measurements of Doppler shifts) are used for localization [12. Vehicle positions are estimated using a least squares approach. To minimize the position uncertainty, a subset of neighbor vehicles are selected as position references via an exhaustive search method.

\section{Non-real-time Centralized Solution}

Our centralized ML solution estimates the position of vehicles by simultaneously capturing a number of spatial and temporal constraints. These constraints are the result of the measurements that the vehicles collect to help them determine their positions. A first set of measurements, yielding the 'spatial' constraints, are inter-vehicle distance estimates with neighbors within communication range, collected at different times by sending an acoustic ping and measuring the time-of-flight, as described by equation 1 . The so-called 'temporal' constraints are estimates of vehicle velocity measurements as gathered by the navigational sensors on-board each vehicle. The temporal constraints are mathematically shown in equation 2. Calculating the collection of vehicle positions over time within the set of spatial and temporal constraints is a complex multi-dimensional estimation problem. To solve it optimally, we have proposed the framework of factor-graphs. Our factor-graph solution efficiently computes the individual pdfs of the position of vehicles at discrete times from the joint pdf of all the unknown positions given measurements (of inter-vehicle distances and motion). The factor-graph itself is a graphical representation of this joint pdf. A detailed discussion of the complete solution and how these 
constraints are represented is available in our prior work [7. The theoretical foundations of this technique have been laid out by Kschischang et. al.[13. Here we only highlight the key ideas behind our centralized and offline algorithm. To do this we use a simplified notation and describe the factor-graph in terms of a dependency graph. The complete factor-graph can be reconstructed from the dependency-graph by replacing each vertex in the dependency graph by its internal representation as discussed in section 3.1 .

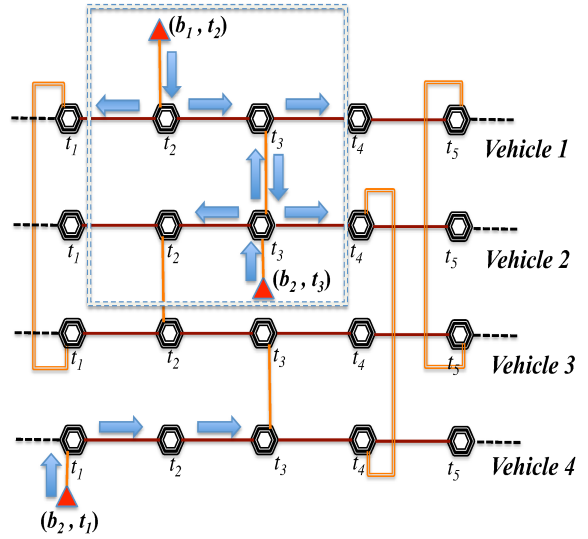

(a)

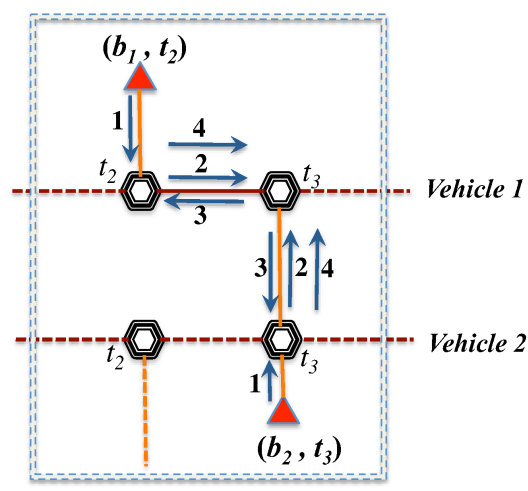

(b)

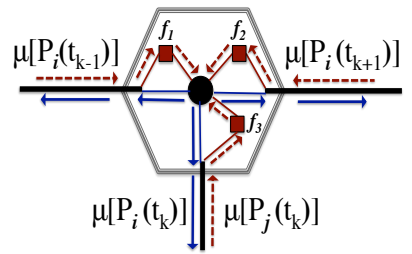

(c)

Figure 1: Centralized non-real-time solution (a) Dependency graph (b) Iterative message passing for position estimation (c) Internal structure of a vertex in the dependency graph for any vehicle $i$ at time $t_{k}$

Figure 1(a) shows the dependency graph for a network of four vehicles. We have shown the graph for only four vehicles to not overload the figure. The structure of the graph can be viewed in terms of four chains stacked vertically. 
Each chain captures the evolution of the unknown position of a vehicle over time. Correspondingly, each vertex in a chain (shown as a hexagon) represents the unknown states (position and velocity) of the vehicle at a particular time instance. A link between any two vertices exists if there is a measurement (or a set of measurements) that relates/constrains those states. Vertices within a chain are linked by measurements of motion, i.e. the temporal constraints. Vertices across chains are linked by measurements of inter-node distance, i.e. the spatial constraints. The triangles represent the position of beacon nodes at any particular time instance. A link with a triangular vertex exists if a measurement of distance was made with the beacon at that time. Note that measurements (of distance and motion) are obtained in real-time and stored on the memory banks of the vehicles. However, the dependency-graph described above is constructed in non-real-time, after all the measurements are available at a central location.

The links in the dependency graph shown in Figure 1(a) establish pathways of information flow across vehicles in the network as well as back and forth along the temporal dimension of each chain in the graph. Initially, position information is only available at the triangular beacon-vertices (we assume beacons are, for example, buoys on the surface equipped with a GPS receiver). The sum-product algorithm that runs on the above described graph generates this information. When we run the sum-product algorithm on the graph, information from the beacon vertices eventually percolates to all the unknown states (vertices) of the factor-graph along the graph edges as depicted in Figure 1(a).

The dependency graph shown above is a simplified notation used for the actual factor-graph. Next, we describe how the factor-graph is obtained from the dependency graph and present the operation of the sum-product algorithm to estimate vehicle positions.

\subsection{Constructing the factor-graph from the dependency graph}

The unknown positions are computed by running the sum-product (SP) algorithm (in non-real-time) on the factor graph. The factor-graph representation 
is obtained from the dependency graph shown in figure 1(a) by replacing each hexagonal vertex by its corresponding internal structure shown in figure 1(c),

To describe the internal structure of each vertex we denote the unknown position of any vehicle $i$ at time $t_{k}$ as $P_{i}\left(t_{k}\right)$. A vertex in the dependency graph is a simplified notation for a state-variable, $P_{i}\left(t_{k}\right)$ and a set of transformation functions $\left(f_{1}, f_{2}\right.$ and $\left.f_{3}\right)$, as shown in Figure $1(\mathrm{c})$, By replacing each vertex in the dependency graph with these elements, the equivalent factor graph is obtained.

The transformation functions $f_{1}, f_{2}$ and $f_{3}$ are computed from the statistical characterization of distance and velocity measurements. We derive these functions from equations relating the unknown positions of vehicles to measurements of distance and velocity. A distance measurement obtained at time $t_{k}$ as a result of communication between two vehicles with positions $P_{i}\left(t_{k}\right)$ and $P_{j}\left(t_{k}\right)$ at that time relates the unknown positions as follows:

$$
z_{d}\left(t_{k}\right)=\left\|P_{i}\left(t_{k}\right)-P_{j}\left(t_{k}\right)\right\|_{2}+\varepsilon_{k}^{R}
$$

where, $z_{d}\left(t_{k}\right)$ is the distance measurement, $\varepsilon$ is the error in distance estimates with any known pdf.

Next, a velocity measurement, $z_{v}\left(t_{k}\right)$ is related to the unknown position from first principles:

$$
z_{v}\left(t_{k}\right)=\frac{1}{\left|t_{k+1}-t_{k}\right|}\left(P\left(t_{k+1}\right)-P\left(t_{k}\right)\right)+\varepsilon_{k}^{V},
$$

$175 \quad$ where, $\varepsilon_{k}^{V}=\left[\varepsilon_{k}^{v_{x}}, \varepsilon_{k}^{v_{y}}\right]^{T}$ is the error in the velocity measurement with known statistics.

The transformation functions $f_{1}, f_{2}$ and $f_{3}$ are defined as follows:

$$
\begin{aligned}
& f_{1}=p\left(P_{i}\left(t_{k}\right) \mid P_{i}\left(t_{k-1}\right), z_{v}\left(t_{k}\right)\right) \\
& f_{2}=p\left(P_{i}\left(t_{k}\right) \mid P_{i}\left(t_{k+1}\right), z_{v}\left(t_{k}\right)\right)
\end{aligned}
$$




$$
f_{3}=p\left(P_{i}\left(t_{k}\right) \mid P_{j}\left(t_{k}\right), z_{d}\left(t_{k}\right)\right)
$$

The exact definitions of the above conditional distributions are obtained from equations 1 and 2 and a statistical characterization of the error terms in these

180 distributed, however any other distributions can be used to define the above conditionals. For completeness we describe the error in distance estimates are Gaussian: $\varepsilon_{k}^{R} \sim U\left(-\sigma_{R \max }, \sigma_{R \max }\right)$. Further, the error in velocity measurements are also Gaussian $\varepsilon_{j}^{v_{x}} \sim U\left(-\sigma_{V \max }, \sigma_{V \max }\right), \varepsilon_{j}^{v_{y}} \sim U\left(-\sigma_{V \max }, \sigma_{V \max }\right)$.

In Figure 1(c), the incoming and outgoing messages are shown by dotted and solid arrows respectively. When the sum-product algorithm runs on the FG constructed using the above notation, the functions, $f_{1}, f_{2}$ and $f_{3}$, operate on the incoming messages $\mu\left[P_{i}\left(t_{k-1}\right)\right], \mu\left[P_{i}\left(t_{k+1}\right)\right]$ and $\mu\left[P_{j}\left(t_{k}\right)\right]$, respectively, to compute independent estimates of the pdf of $P_{i}\left(t_{k}\right)$, as per equation 6. The outgoing message $\mu\left[P_{i}\left(t_{k}\right)\right]$ is computed as per equation 7 .

\subsection{Operation of the sum-product algorithm}

The sum-product algorithm is essentially an iterative message passing algorithm. At each iteration, every vertex in the graph estimates the pdf of its own states based on incoming messages. (A vertex encapsulates the unknown states and a set of transformation functions that operate on incoming messages to compute these estimates as described in section 3.1). The pdfs computed for each vertex are then passed on as messages to all neighbor vertices.

At each time instance the SP algorithm does two things. First, the statevariables are estimated by intersecting the individual estimates from its function node neighbors, equation 7 . Next the SP estimates the function nodes by marginalizing the local likelihood function of each state-variable, equation 6. This is repeated until convergence. Note that there are two very distinct notions of time here: the time represented by states in the graph (which is the time evolution of the network we are trying to estimate) and the time post-facto during which we run our algorithm on a central location (the running time). 


$$
\mu_{f-x}(x)=\sum_{\sim\{x\}}\left(f(X) \prod_{y \in(f) \backslash\{x\}} \mu_{y-f}(y)\right)
$$

where the summary operation is defined as:

$$
\begin{aligned}
& \sum_{\sim x_{1}} f\left(x_{1}, x_{2}, x_{3}\right)=\sum_{x_{2} \in A_{2}} \sum_{x_{3} \in A_{3}} f\left(x_{1}, x_{2}, x_{3}\right) \\
& \mu_{x-f}(x)=\prod_{h \in n(x) \backslash f} \mu_{h-x}(x)
\end{aligned}
$$

where, $X$ is the set of arguments of function node $f ; n(w) \backslash\{x\}$ denotes the set of neighbors of a given node $w$ on the graph excluding the node $x$.

The key point in the above described factor-graph solution is that to effectively fuse position information, messages have to be passed a number of times across each of the links of the graph as depicted for a small section of the graph in Figure 1(b). The solution above runs offline and centrally after the actual sensing mission is over. Next, we will explore if and how we can modify this solution to tackle the problem of real-time tracking in underwater networks.

\section{Real-time Tracking}

\subsection{Centralized real-time tracking}

In this first subsection, we try to answer the question: what is the fundamental difference between a non-real-time and a real-time estimation of vehicle positions? Here we still assume that computation can run in a central location. While in our offline non-real-time solution (discussed in section 3 ) the factorgraph was constructed in one shot using all measurements made when vehicles were underwater, here we propose a dynamic factor-graph solution that evolves in time to (a) incorporate new states (position and velocity), (b) incorporate estimates of motion and distance with neighbor vehicles as they become available, and (c) limits the use of future information in estimating positions.

We are interested in knowing the positions of vehicles at discrete times $\delta$ apart. At any time $t$, the FG consists of $N$ chains of vertices representing 


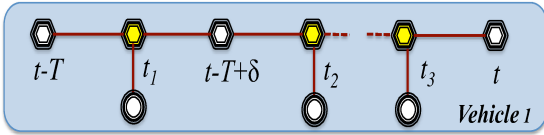

(a)

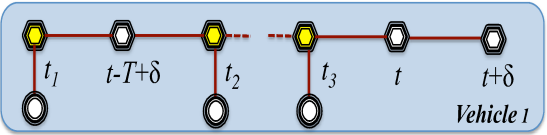

(b)

Figure 2: Evolution of dynamic FG (a) FG at time $t$ (b) FG at time $t+\delta$

the unknown states of all vehicles until that time. A new vertex is added to the tail of each chain every $\delta(\mathrm{s})$ as depicted in Figure 2. In addition, when a measurement of distance becomes available between two vehicles $i$ and $j$, due to a communication event at time $t$, a new vertex is inserted to the $i^{\text {th }}$ and $j^{\text {th }}$ chain in the graph corresponding to time $t$ and a link is created between them. Each new state is also appropriately linked to previous states in a chain using measurements of motion. Whenever the FG is updated with a new state or measurement, the SP algorithm is run on the graph to compute updated estimates of all states. To obtain position estimates in real-time, the pdf of any state in the FG corresponding to time $t$ is recorded at that time, before future measurements become available. In principle we can estimate the vehicle positions semi real-time, by limiting the extent of future information to a time window $\tau$. Essentially, estimates corresponding to any time $t$ are obtained from the FG at $t+\tau$. This is generally referred to as smoothing. Such a solution would be suitable for applications that can tolerate some delay in obtaining position estimates.

We implemented our centralized real-time tracking solution in the NS2 simulator [14. The simulation set up is described later in Section 4.3 with parameters given in Table 1. In this section we evaluate the localization performance as a function of the smoothing delay, $\tau$. Figure 3 shows the cdf of the RMS error in position estimates for different values of $\tau$. In the figure, the performance of the offline solution corresponds to $\tau=\infty$ and that of the real-time tracking corresponds to $\tau=0$. Notice the loss in performance as we go from a non-real-time solution to a real-time one. This is due to the fact that the real-time solution does not use any future measurements for estimating the unknown states. How- 
ever, as time elapses information flows backward (along the horizontal links of the graph) to refine past position estimates. Figure 3 also shows the gain in localization performance as the smoothing parameter, $\tau$ is increased. We observe that the performance of the semi-real-time tracking coincides with that of the non-real-time solution for $\tau=75 \mathrm{~min}$ which is a fraction of the total simulation time of 8 hours.

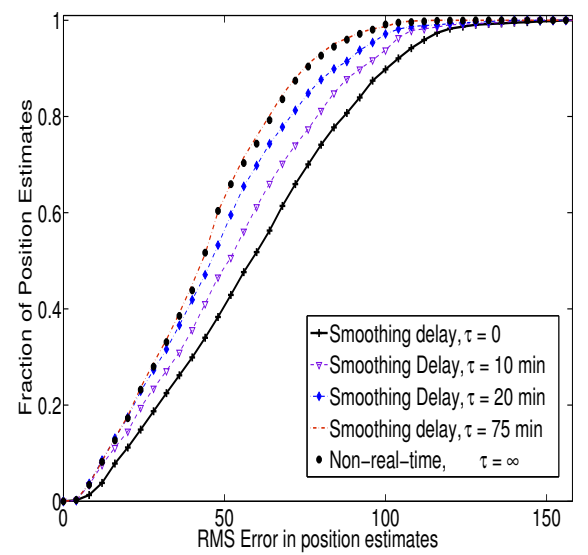

Figure 3: Cumulative Distribution Function (CDF) of the RMS error as a function of the smoothing parameter, $\tau$. Each curve in the plot shows the fraction of position estimates (indicated by the y-axis), computed for all vehicles for the entire simulation duration, with RMS error less than a given threshold (indicated by the $\mathrm{x}$-axis)

While the simulation results suggest that we can get better localization accuracy by using estimates slightly in the past, in reality we would still need to take into account the fact that the vehicle has moved during the interval $\tau$. Therefore, past (refined) estimates have to be interpolated to the present time. However, this is what our tracking algorithm does in an optimal way using navigational measurements. Therefore, applications that are interested in knowing where a vehicle is 'now' in order to make location-based decisions cannot leverage from the parameter $\tau$. This is true for most applications such as navigation and control, routing and so on. However, refining past estimates would be useful for annotating data samples with position information and batch processing 
them in semi-real time. The smoothing delay is one of the parameters that inherently limits the performance of a real-time and distributed solution. In

in a distributed setting, a vehicle has to share updates about all its states (past and current) with those vehicles that had obtained a distance measurement to 


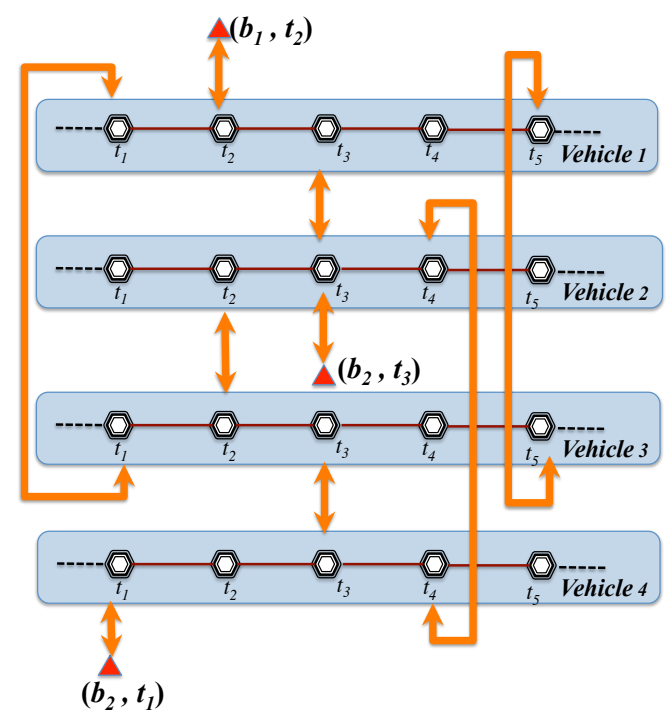

Figure 4: Decomposed view of centralized dependency graph

it. This is equivalent to passing messages over all links of the centralized FG. However, in a distributed setting, messages can only be passed over the active communication links of the physical acoustic network. The problem is that since vehicles are moving, past states cannot be shared with those vehicles that have gone out of communication rang $\emptyset^{1}$. We have depicted the problem that arises in the distributed case in Figure 5(a) The figure shows the network topology for three vehicles $A, B$ and $C$ at times $t_{1}$ and $t_{2}$. At time $t_{1}, B$ communicates with $A$ and shares its current position state, $P_{B}\left(t_{1}\right)$. Vehicle $A$ also estimates its distance to $B$ at the same time and incorporates this information in its factor-graph as shown in Figure $5(\mathrm{c})$. Later at time $t_{2}, B$ gets new position information from vehicle $C$, which improves its past estimate at $t_{1}$. Now in a centralized solution, this updated information would have been passed to the subgraph of vehicle $A$,

\footnotetext{
${ }^{1}$ Note that a similar problem exists in the centralized formulation because all measurements have to be routed to a single location, while there may be times when no route is available. However, in our simulations we assume that measurements are always available at a single location for centralized tracking. This was done to carry out a logical study of the factors that affect the performance of the distributed solution.
} 
scenario this is not possible because $A$ and $B$ are no longer in communication range.
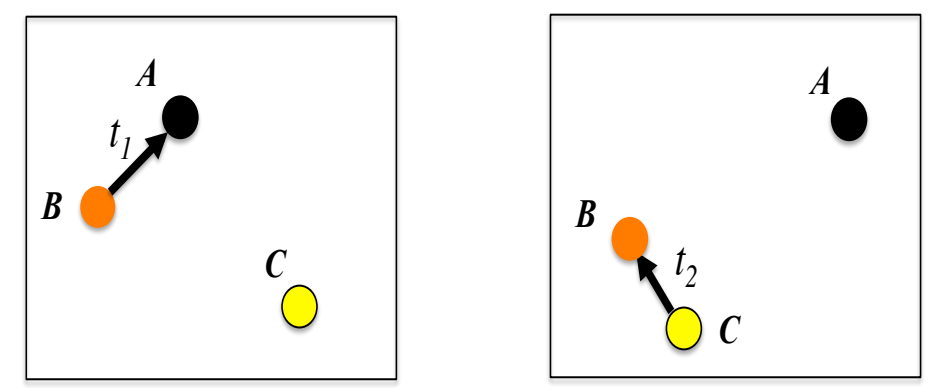

(a)

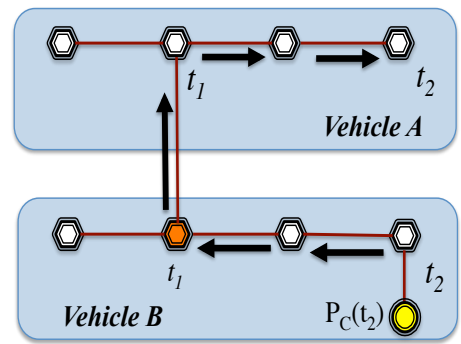

(b)

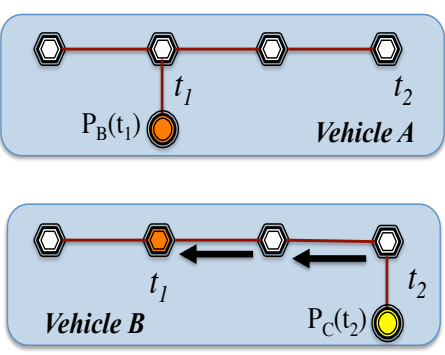

(c)

Figure 5: (a) Network topology at times $t_{1}$ and $t_{2}$. FG at time $t_{2}$ for (b) Centralized tracking (c) Distributed tracking

To achieve the best localization performance in the distributed case, we propose that vehicles share their states with those that are multiple hops away. To do so they periodically broadcast their current and past position states as well as forward the states of other vehicles. This addresses the problem described in Figure 5 because when $B$ improves its past estimate, $P_{B}\left(t_{1}\right)$ at a later time $t_{2}$, it routes this improved estimate to $A$. The limitation of this scheme compared to the centralized solution is that updates cannot be shared with vehicles that are not reachable via multihop. Nevertheless it gives the best possible real-time distributed localization performance (by construction) and serves as a performance baseline. In the remainder of the paper we will propose ways to minimize 
the energy overhead of the distributed tracking.

\subsection{Distributed tracking with local information sharing}

325 However, since underwater acoustic communication is resource intensive and the vehicles are energy-constrained, we now consider ways to make our distributed solution more practical. To limit the communication, we propose schemes that only use local information obtained from communication with immediate neighpast position states with its immediate neighbors. In the second scheme we further reduce the amount of information transmitted by vehicles by having them periodically communicate only their current position state to their immediate neighbors. We next compare the performance of the tracking solutions presented so far using the simulation setup described in [8].

Simulation setup: We implemented all the elements of our real-time, centralized and distributed FG tracking in NS2 using the NSMiracle extensions for simulating underwater network protocols [14]. We simulated a network with 8 mobile vehicles and 1 beacon. The vehicles were placed in a $2 \mathrm{~km} \mathrm{x} 2 \mathrm{~km}$ area. We used the mobility model proposed in [7] where vehicles follow smooth curves Vehicles transmitted localization updates every $T_{L O C}(\mathrm{~s})$. While our proposed information sharing schemes can be used with any MAC protocol, we chose a TDMA MAC. The simulation parameters are summarized in Table 1.

345 of our offline solution (see section 3), real-time centralized (see section 4.1), real-time distributed (best case) (see section 4.2) and the real-time distributed tracking solutions with local information sharing (described earlier in this section). The CDF of RMS error in position estimates for the different tracking formance occurs when we go from an offline solution to a real-time one.

While there is some benefit in forwarding updates over multiple hops, the 
Table 1: Simulation Parameters

\begin{tabular}{|c|c|}
\hline Tracking granularity, $\delta$ & $2 \mathrm{mins}$ \\
\hline Localization period, $T_{L O C}$ & 8 mins \\
\hline Simulation duration, $T_{\text {sim }}$ & 8 hours \\
\hline Number of vehicles, $N_{U}$ & 8 \\
\hline Number of beacons, $N_{B}$ & 1 \\
\hline Area of deployment, $A$ & $2 \mathrm{~km} \times 2 \mathrm{~km}$ \\
\hline Maximum depth of deployment, $D$ & $100 \mathrm{~m}$ \\
\hline Maximum transmission range, $R$ & $500 \mathrm{~m}$ \\
\hline Average speed of vehicles, $\bar{v}$ & $0.5 \mathrm{~m} / \mathrm{s}$ \\
\hline Bytes used to represent pdfs internally, $N_{\text {internal }}$ & 512 \\
\hline Bytes used to communicate pdfs, $N_{\text {comm }}$ & 18 \\
\hline
\end{tabular}

energy overhead of doing so is substantial. However, we can come very close to the best case distributed performance by sharing past estimates with immediate neighbors. For these simulations, the results suggest that if performance is the key requirement with nominal constraints on energy a good compromise solution may be to share current and past states with immediate neighbors. However, if energy is of primary concern, then periodically sharing only the current state with immediate neighbors is sufficient.

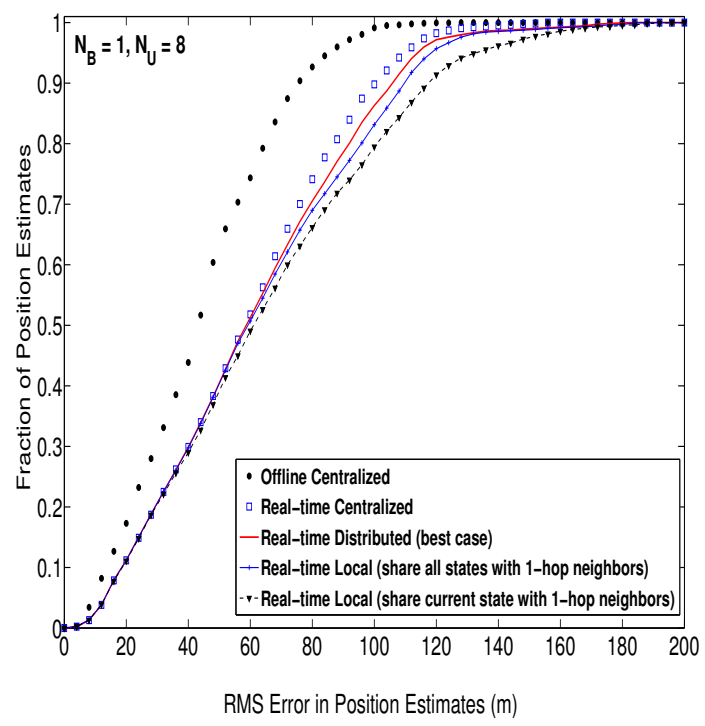

Figure 6: CDF of RMS error for proposed information schemes

To get a better understanding of the relative benefit of the different schemes, we evaluated their performance when the number of beacons, $N_{B}$ and number 
of vehicles $N_{U}$ were varied. Later in section 6, we provide a more in-depth discussion of how this affects the localization performance.

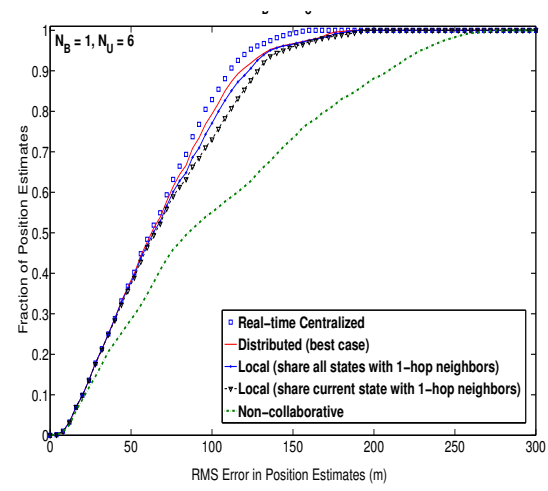

(a)

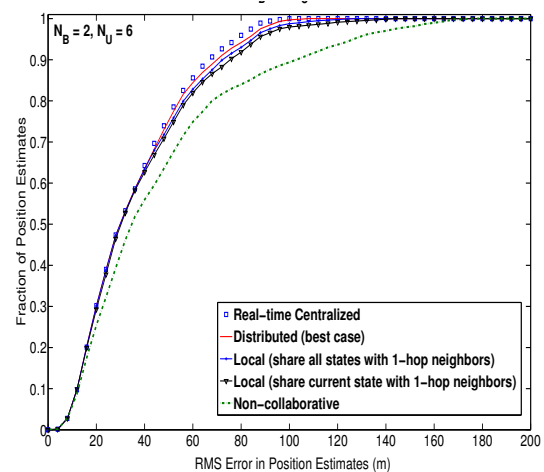

(c)

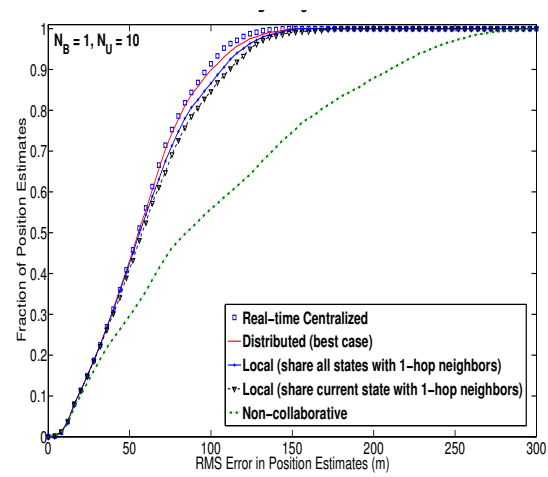

(b)

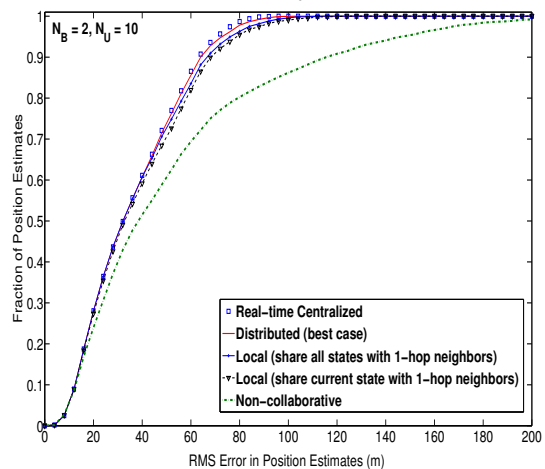

(d)

Figure 7: Performance of real-time centralized, real-time distributed (best case) and real-time distributed tracking solutions with local information sharing for networs with (a) one beacon and 6 unknown nodes, (b) one beacon and 10 unknown nodes, (c) two beacons and 6 unknown nodes, (d) two beacons and 10 unknown nodes

Figure 7 shows the CDF of the RMS error in position estimates for the

different tracking solutions as well for the non-collaborative case where vehicles only use measurements with beacons for position estimation. These results show that there is significant gain in localization accuracy from inter-vehicle collaboration. Further, this gain increases with the number of vehicles because of the 
additional constraints obtained from inter-vehicle distance estimates. Among hicles improves localization performance when there is only one beacon in the network. For the networks with one beacon, when the network connectivity is very low $\left(N_{U}=6\right)$, sharing past states with only immediate neighbors almost achieves the best case performance. However, when the connectivity improves formance. This is because under conditions of low network connectivity vehicles that would benefit from past position updates are not reachable via multihop. This also explains the performance difference between the real-time centralized and the best case distributed solutions for $N_{B}=1$ and $N_{U}=6$. However, we observed that when the number of beacons is increased $\left(N_{B}=2\right)$, the performance of the different information sharing schemes almost overlap, showing little gain in sharing past estimates over single or multiple hops. Nevertheless we still observe a significant gain over the non-collaborative case.

There may be times when the best performance is needed, which requires vehicles to share updates about past position estimates over single and multiple hops. However, we observed that in most cases, it is sufficient to periodically share only the current position estimate with neighbors.

\section{Implementation Details}

\subsection{Message Representation}

390

The sum-product algorithm operates efficiently on the pdf of discrete random variables while both position and velocity are most naturally defined over continuous space. To address this problem, we approximate the true pdf of states by piece-wise constant distributions that are uniformly weighted over $2 \mathrm{D}$ grids [7. We have shown in our prior work that if these distributions are binary weighted, the entire sum-product algorithm can be implemented in binary logic which significantly reduces the computation overhead [7]. 
To enable vehicles to control their energy consumption without significantly deteriorating their localization accuracy we propose to decouple the representation used when communicating pdfs over the wireless channel from that used internally by vehicles. A more in depth description of the proposed solution and how pdfs of different granularities can be compared can be found in our prior work [8. The key point is that we can adapt the number of bytes used to communicate pdfs over the wireless channel, without significantly deteriorating the performance of the algorithm. This is summarized in Figure 8 where we compare the localization performance for various values of $N_{\text {comm }}$, the number of bytes used to represent communicated pdfs and $N_{\text {internal }}$, the number of bytes used to represent pdfs local to the nodes. The conclusion we can draw from this figure is that we maintain sufficient accuracy when only transmitting 18 bytes of data for each pdf and maintaining an internal pdf of 512 bytes. This low communication overhead is key for a practical implementation of this system. There is a trade off between the number of grid points used to represent pdfs internally, $N_{\text {internal }}$, and the computation overhead of the algorithm. The number of grid points is inversely related to the size of each grid. In the next section we will discuss why and how the grid size adversely affects the accuracy of the algorithm and propose an implementation to counter this effect as much as possible under practical constraints.

\subsection{Implementation of the Factor Graph for effective information fusion}

While uniformly weighted piecewise constant distributions minimize the computation and energy overhead of the sum-product algorithm, they deteriorate the accuracy of position estimates by introducing rounding errors. In this section we present why these errors are introduced and provide an approach that minimizes their effect.

The local factor graph of each vehicle essentially fuses distance estimates to neighbor vehicles, obtained at different times, to compute the pdf of all the states specified by the vertices of the graph. Each distance measurement (in combination with an estimate of the position of the neighbor vehicle) provides 


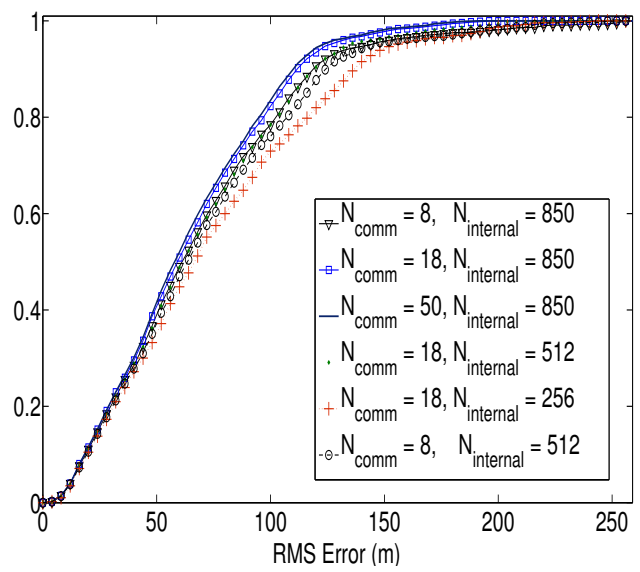

Figure 8: CDF of RMS error in position estimates as a function of the number of bytes used to communicate each message, $N_{\text {comm }}$ and the number of bytes used to represent the message internally, $N_{\text {internal }}$

an independent estimate of the position of the vehicle at the time that it was obtained. In principle the measurements of the vehicle's motion allow these estimates to be translated to any past or future time, $t$ where they are fused.

${ }_{430}$ The translation in time is mathematically given by

$$
P\left(t \mid z_{i}\right)=P\left(t_{i} \mid z_{i}\right)+\bar{v}\left(t_{i}, t\right)\left|t_{i}-t\right|+\varepsilon_{v}\left|t_{i}-t\right|
$$

where $z_{i}$ denotes the distance estimate obtained at time $t_{i}, P\left(t_{i} \mid z_{i}\right)$ denotes the estimate of the position of the vehicle at time $t_{i}$ due to the distance measurement obtained at $t_{i}$ alone, $\bar{v}\left(t_{i}, t\right)$ is the estimate of the vehicle's average velocity in the interval $\left(t_{i}, t\right)$ and $\varepsilon_{v}$ is the error in velocity measurements.

As the error term in the above equation shows there is additional uncertainty in the translated position estimate $P\left(t \mid z_{i}\right)$ since measurements of the vehicle's motion are not exact.

In practice, the translation step given by equation 8 is performed numerically by the SP algorithm. This introduces a rounding error every time a transla440 tion is performed because the true pdf is approximated by the binary weighted piecewise constant pdfs (that were described in the previous section). In effect the true pdf of any state is smeared every time information is passed from one 
state in the graph to an adjacent state. The extent of smearing at each stage depends on the grid size of the representation. Further, the rounding errors introduced due to grid size have a cumulative effect when information available in one state has to pass through many intermediate states (that do not provide additional information) in order to be merged with information available in a different state in the graph.

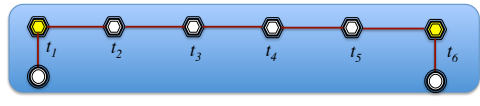

Figure 9: Factor graph with 4 intermediate states between $t_{1}$ and $t_{6}$

To further elucidate the effect of intermediate states and grid size on roundwhich increases the computation overhead. This problem becomes specially pronounced when distance measurements become available very intermittently compared to the tracking granularity, $\delta$. To address this issue we propose an 
alternate implementation of the factor-graph that was earlier presented in Sec-

We now evaluate the performance of our proposed FG implementation versus the one where all the unknown states are estimated in one shot. In order to isolate the effect of intermediate empty states on the localization accuracy from 
all other parameters that affect performance, we simulated a scenario where only one vehicle was tracked using distance estimates from beacons. A measurement of distance was made with a beacon every 36s. Three beacons were used for tracking. The vehicle position was estimated every 3s. Keeping the maximum number of grid points used to represent the pdf of position estimates the same, we compared the RMS error in position estimates obtained over 20 minutes of simulation time using the FG implementation shown in Figure 2 and the proposed implementation shown in Figure 10. The cumulative distribution function (cdf) of the RMS error in estimates for the two implementations is shown in Figure 11(a) and the RMS error versus time is shown in Figure 11(b), From these results we observe that the localization accuracy improves significantly using our proposed implementation.

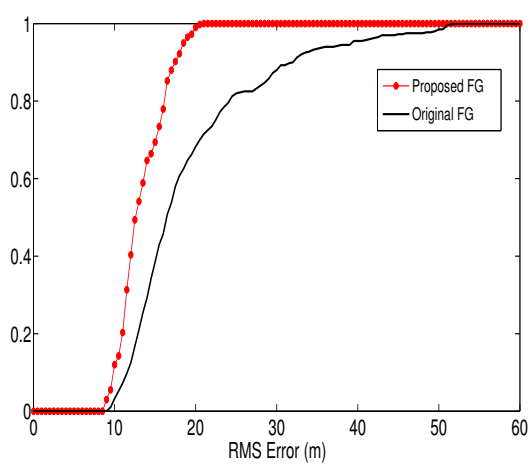

(a)

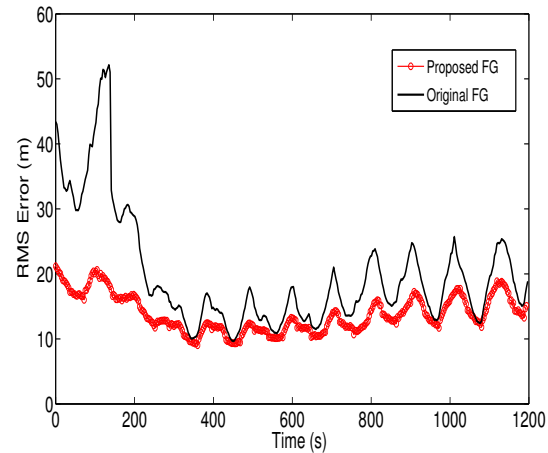

(b)

Figure 11: Performance of proposed FG implementation vs original (a) Cumulative Distribution function of RMS Error (b) RMS Error versus time

will conceptually evolve as described in section 4 however we implement it as explained in this section. In addition, the grid size used to represent pdfs internally is kept small by using signifcantly larger number of bytes to represent these pdfs internally compared to the number of bytes used to communicate

these pdfs between vehicles. This essentially translates to choosing a much 
larger value for the parameter $N_{\text {internal }}$ compared to the parameter $N_{\text {comm }}$.

\section{Deployment Considerations}

For the simulations in this section we compare non-collaborative localization to sharing the current state with a 1-hop neighbor (shortened to "Collaborative"

\subsection{Dependence on Proximity to Beacon}

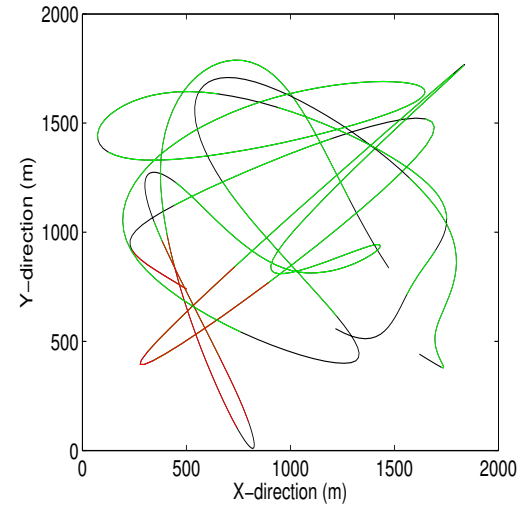

(a)

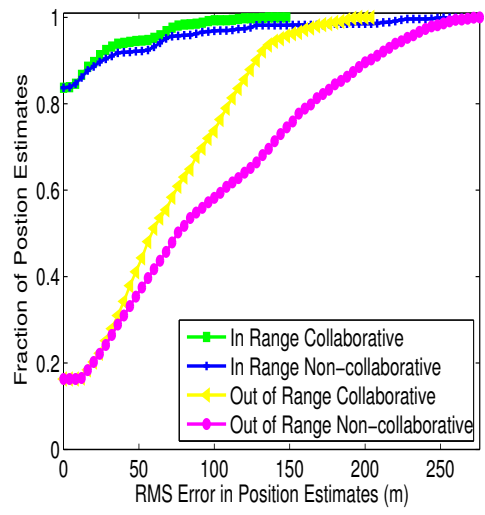

(b)

Figure 12: (a) Trajectory of one of the nodes under evaluation with regions indicating direct communication with beacon (red), regions indicating communication with only other unknown nodes (green) and regions of no communication (black) (b) CDF of RMS error comparing the difference between the error when nodes are in range of the beacon versus when they are out of range of the beacon

Since position information always percolates into the network starting at a beacon, one of the factors that naturally determines the error in position 
estimates is the proximity of nodes to beacons. In this section we quantify the there is not much gain from using collaboration. However, if it is out of range of beacons significant gains can be obtained by leveraging the localization information of other nodes. Notice that this gain can only occur when collaboration 
provides the nodes good information, i.e. the collaboration nodes are in communication with a beacon. While the specific trajectory is not important, what is important is whether or not a node can receive good information from other nodes when they are collaborating. We will see examples later where sharing bad information can actually hurt the localization performance of a specific node.

The results support what we intuitively expect: the error in position estimates is least when a node is in the direct communication range of a beacon. Additionally, we observe that both in the case of collaborative and noncollaborative localization, there is significant disparity in the error when nodes are within the range of the beacon compared to when they are not. This indicates that a key factor in determining the position accuracy of a node is whether or not it is within the communication range of a beacon and the benefit of direct communication with a beacon outweighs the gains from inter-node collaboration.

Based on the above findings we would like to develop the importance of a node periodically receiving localization information from a beacon. Addressing this question relates to strategizing the placement of beacons in a network, as the number of available beacons is increased.

\subsection{First Hand vs Second Hand Information}

575 In this section we consider the following question: If the number of beacons available for tracking is increased, would it be better to distribute them uniformly or non-uniformly over the area of deployment?

We consider the above question because when the spatial distribution of beacons is not uniform we expect the error of a subset of the nodes to be minimized. In principle these nodes can then localize other nodes in the network that have less frequent (or no access) to beacons. On the other hand when the beacons are uniformly distributed in the network we expect all nodes to achieve approximately the same level of accuracy. Since most nodes do not have an information advantage over others, we would also expect little gains 


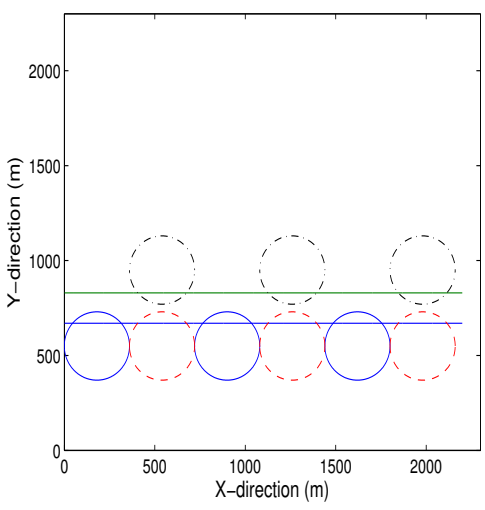

(a)

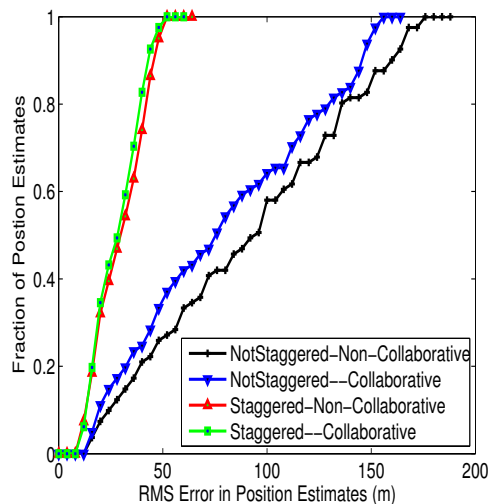

(b)

Figure 13: Experiments describing hearing a beacon vs receiving location information from another unknown (a) Non-Staggered: (red dotted line for beacon range) the top node cannot communicate with the beacons Staggered: (black .- line for beacon ranges) the top node periodically communicates with beacons (b) CDF of error in position estimates for collaborative and non collaborative localization schemes.

from collaboration in such a scenario.

To investigate the above conjectures we considered two simulation scenarios shown in Figure 13(a) consisting of six stationary beacons and two unknowns that are always within the communication range of each other. In one of the scenarios all six beacons were arranged linearly in a non-staggered fashion such that the bottom node (whose trajectory is shown in blue) is always within the range of a beacon and the top node never hears a beacon. In the second scenario the beacons were placed in a staggered fashion, such that the bottom (blue) track intersects the communication range of three of the beacons and the top (green) track intersects the communication range of the other three beacons. We considered the localization error of the top (green) track to compare the benefit of receiving information from a beacon and a neighboring node (the staggered beacon arrangement) to the case where we only receive information from a neighboring node who receives information from twice as many beacons (the non-staggered beacon arrangement). The cdf of the RMS error in the position 
non-staggered arrangement where the top node never hears a beacon there is noticeable gain from communicating with the bottom node.

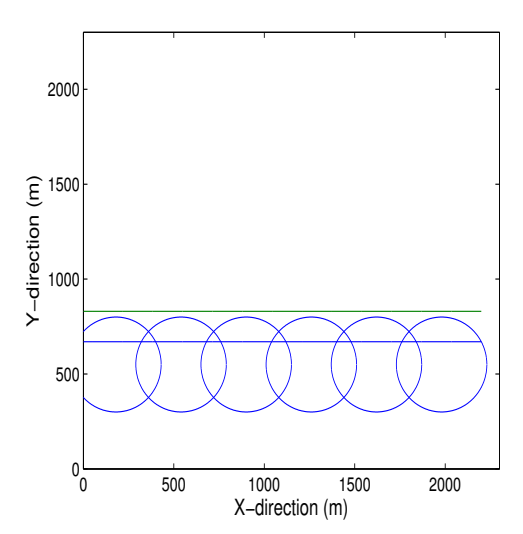

(a)

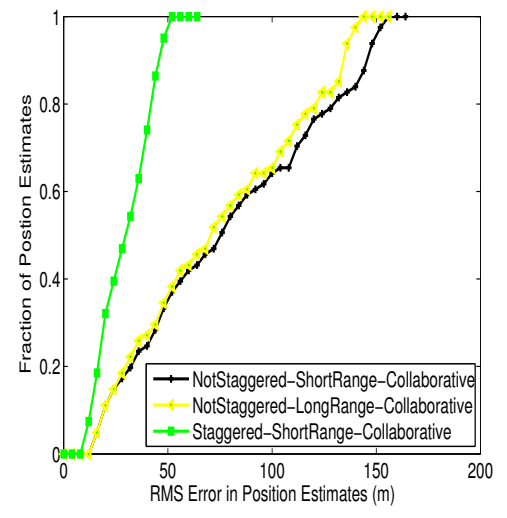

(b)

Figure 14: (a) Non-Staggered beacon arrangement with increased beacon transmission range (blue .- line for beacon ranges) (b) results plotted for the top (green) trajectory

Additionally, we considered the case shown in Figure 14(a) where the beacon range is extended so that the bottom node intermittently receives updates from two beacons at a time to further reduce its error. In this case there is an additional improvement in the accuracy of the top track when the tracking is done collaboratively as shown in Figure 14(b). This indicates that we can increase the location accuracy of both nodes by increasing the number of beacons used to localize one of them. However, this gain is clearly inferior to allowing the top node to receive location information from a beacon directly. 


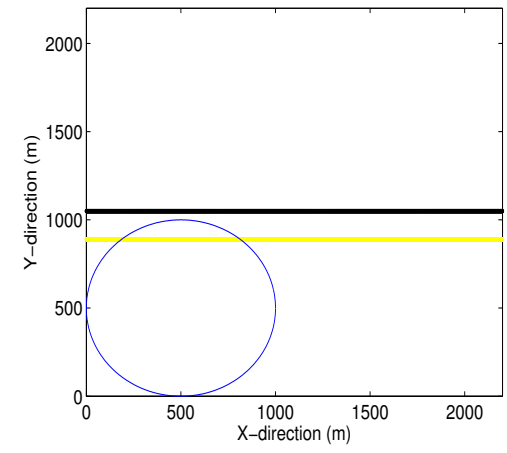

(a)

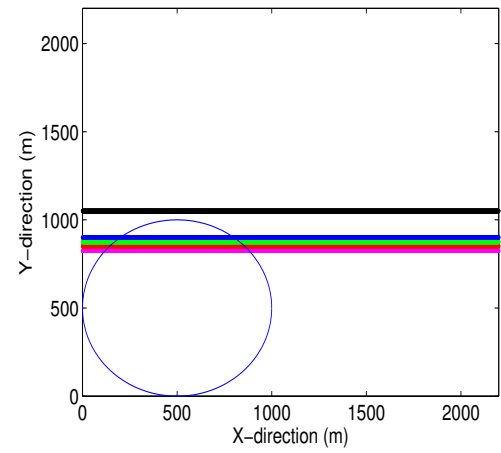

(b)

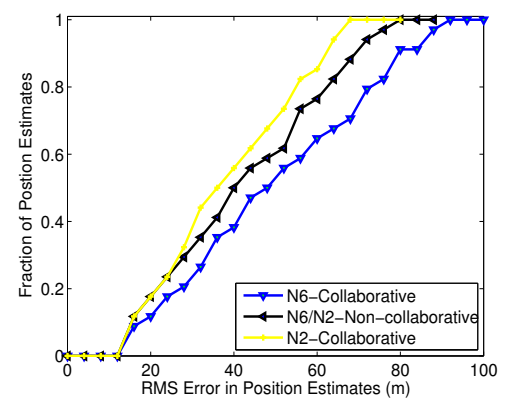

(c)

Figure 15: Trajectories quantifying the results of sharing redundant information (beacon range shown in blue ellipse) (a) 2 node network (b) 6 node network (c) Results- CDF of RMS error in position estimates: note that the performance of the non-collaborative tracking is the same for all cases

\subsection{Redundant Information}

In this section we would like to understand how much collaboration is really useful and when. We evaluate whether a node can improve its estimate when communicating with nodes traveling in similar trajectories. To do this we consider the networks of Figure 15, where one node is never in communication range of a beacon while the rest of the nodes are in range of the beacon at some point during the simulation. In the first case, Figure $15(\mathrm{a})$, there are 2 nodes following similar trajectories. The bottom node is in range of the beacon 
for a portion of the simulation while the top node can only receive localization errors are a result of representing the estimated pdfs using only a finite and nominal number of grid points. As such this effect would not come into play if the pdfs could be represented with infinite accuracy. The above simulation 
scenario was specifically chosen to show that when all nodes in the network have poor estimates of their positions, sharing redundant information does not provide any benefit, but rather can hurt the localization accuracy of the nodes due to practical limitations in the implementation of the algorithm.

Our simulation results show that direct communication with beacons, network connectivity and inaccuracies in the position estimates limit the scenarios where the proposed collaborative algorithm outperforms a non-collaborative approach. To summarize our conclusions:

1. The localization accuracy of any node is primarily determined by the extent of time it is in direct communication with beacons. If multiple beacons are available it is more beneficial to distribute them in such a way that all vehicles have an equal opportunity to periodically hear at least one beacon

2. If a node is in the range of beacons, there is not much gain from using collaboration. However, if it is out of range of beacons significant gains can be obtained by leveraging the position estimates of other nodes.

3. Collaboration is beneficial when nodes do not have direct communication with beacons, however, due to practical limitations of the factor-graph algorithm, nodes with large and growing position errors should refrain from sharing their estimates with others.

\section{Conclusion}

In this paper we reviewed a low overhead collaborative tracking solution for underwater mobile networks that estimates the location of vehicles in real-time and in a distributed fashion. We identified key factors that fundamentally limit the performance of real-time (centralized and distributed) solutions, quantifying their effects via simulations. We also showed that in certain scenarios, vehicles need to share information over multiple hops for best case performance. However, in our proposed scheme they only use information that is obtained locally. We further tested the collaborative localization framework against different motion models and concluded that in a network where nodes follow more 
predictable trajectories, the localization performance can be most significantly

[1] J. Jaffe, C. Schurgers, Sensor networks of freely drifting autonomous underwater explorers, in: Proceedings of the 1st ACM International Workshop on Underwater Networks, WUWNet '06, ACM, New York, NY, USA, 2006, 
pp. 93-96. doi:10.1145/1161039.1161058.

URL http://doi.acm.org/10.1145/1161039.1161058

[2] M. F. Fallon, G. Papadopoulos, J. J. Leonard, N. M. Patrikalakis, Cooperative auv navigation using a single maneuvering surface craft, The International Journal of Robotics Research (2010) 0278364910380760.

[3] G. Rui, M. Chitre, Cooperative positioning using range-only measurements between two auvs, in: OCEANS 2010 IEEE - Sydney, 2010, pp. 1-6. doi: 10.1109/OCEANSSYD . 2010.5603615

${ }_{720}^{\text {n }}$ [4] M. Erol, L. F. M. Vieira, M. Gerla, Localization with dive'n'rise (dnr) beacons for underwater acoustic sensor networks, in: Proceedings of the Second Workshop on Underwater Networks, WuWNet '07, ACM, New York, NY, USA, 2007, pp. 97-100. doi:10.1145/1287812.1287833. URL http://doi .acm.org/10.1145/1287812.1287833

725 [5] A. Teymorian, W. Cheng, L. Ma, X. Cheng, X. Lu, Z. Lu, 3d underwater sensor network localization, Mobile Computing, IEEE Transactions on 8 (12) (2009) 1610-1621. doi:10.1109/TMC. 2009.80

n [6] Z. Zhou, J.-H. Cui, S. Zhou, Efficient localization for large-scale underwater sensor networks, Ad Hoc Networks 8 (3) (2010) 267 - 279. doi:http://dx.doi.org/10.1016/j.adhoc.2009.08.005

口 URL http://www.sciencedirect.com/science/article/pii/ S157087050900081X

n [7] D. Mirza, C. Schurgers, Collaborative tracking in mobile underwater networks, in: Proceedings of the Fourth ACM International Workshop on 735 UnderWater Networks, WUWNet '09, ACM, New York, NY, USA, 2009, pp. 5:1-5:8. doi:10.1145/1654130.1654135. URL http://doi .acm.org/10.1145/1654130.1654135

q [8] D. Mirza, C. Schurgers, R. Kastner, Real-time collaborative tracking for underwater networked systems, in: Proceedings of the Seventh ACM Inter- 
national Conference on Underwater Networks and Systems, WUWNet '12,

ACM, New York, NY, USA, 2012, pp. 3:1-3:8. doi:10.1145/2398936. 2398940 .

URL http://doi.acm.org/10.1145/2398936.2398940

[9] Z. Zhou, Z. Peng, J.-H. Cui, Z. Shi, A. Bagtzoglou, Scalable localization with mobility prediction for underwater sensor networks, Mobile Computing, IEEE Transactions on 10 (3) (2011) 335-348. doi:10.1109/TMC. 2010. 158.

[10] H.-P. Tan, R. Diamant, W. K. Seah, M. Waldmeyer, A survey of techniques and challenges in underwater localization, Ocean Engineering 38 (14) (2011) 1663-1676.

[11] M. Erol-Kantarci, S. Oktug, L. Vieira, M. Gerla, Performance eval-

a uation of distributed localization techniques for mobile underwater acoustic sensor networks, Ad Hoc Networks 9 (1) (2011) 61 - 72. doi:http://dx.doi.org/10.1016/j.adhoc.2010.05.002 URL http://www.sciencedirect.com/science/article/pii/ S1570870510000612

[12] B. Chen, D. Pompili, Uncertainty-based localization solution for underice autonomous underwater vehicles, in: Proceedings of the Sixth ACM International Workshop on Underwater Networks, ACM, 2011, p. 13.

[13] F. Kschischang, B. Frey, H.-A. Loeliger, Factor graphs and the sum-product algorithm, Information Theory, IEEE Transactions on 47 (2) (2001) 498519. doi:10.1109/18.910572.

[14] N. Baldo, F. Maguolo, M. Miozzo, M. Rossi, M. Zorzi, Ns2-miracle: A a modular framework for multi-technology and cross-layer support in net765 work simulator 2, in: Proceedings of the 2Nd International Conference on Performance Evaluation Methodologies and Tools, ValueTools '07, ICST 
(Institute for Computer Sciences, Social-Informatics and Telecommunications Engineering), ICST, Brussels, Belgium, Belgium, 2007, pp. 16:1-16:8. URL http://dl . acm.org/citation.cfm?id=1345263.1345284

\section{Appendix}

This paper is an extension of our previous work on collaborative tracking [8], published in the International Conference on Underwater Networks and Systems (WUWNet'12). In this paper we have made two key additions compared to the conference version:

1. An in depth discussion of the implementation of the Factor-Graph Algorithm, presented in section 5.2

2. Deployment considerations that can significantly impact the localization accuracy of the network, presented in Section 6. In this section we have identified and analyzed scenarios where our collaborative approach shows significant gains, as well as those that highlight the limitations of our approach

The materials presented in both sections 5.2 and 6 have not appeared in any previous publication. 